\title{
CANTOR SPECTRUM FOR SCHRÖDINGER OPERATORS WITH POTENTIALS ARISING FROM GENERALIZED SKEW-SHIFTS
}

\author{
ARTUR AVILA, JAIRO BOCHI, AND DAVID DAMANIK
}

\begin{abstract}
Авstract. We consider continuous SL $(2, \mathbb{R})$-cocycles over a strictly ergodic homeomorphism which fibers over an almost periodic dynamical system (generalized skew-shifts). We prove that any cocycle which is not uniformly hyperbolic can be approximated by one which is conjugate to an $\mathrm{SO}(2, \mathbb{R})$-cocycle. Using this, we show that if a cocycle's homotopy class does not display a certain obstruction to uniform hyperbolicity, then it can be $C^{0}$-perturbed to become uniformly hyperbolic. For cocycles arising from Schrödinger operators, the obstruction vanishes and we conclude that uniform hyperbolicity is dense, which implies that for a generic continuous potential, the spectrum of the corresponding Schrödinger operator is a Cantor set.
\end{abstract}

\section{Statement of the Results}

Throughout this paper we let $X$ be a compact metric space. Furthermore, unless specified otherwise $f: X \rightarrow X$ will be a strictly ergodic homeomorphism (i.e., $f$ is minimal and uniquely ergodic) that fibers over an almost periodic dynamical system. This means that there exists an infinite compact abelian group $\mathbb{G}$ and an onto continuous map $h: X \rightarrow \mathbb{G}$ such that $h(f(x))=h(x)+\alpha$ for some $\alpha \in \mathbb{G}$. Examples of particular interest include:

- minimal translations of the $d$-torus $\mathbb{T}^{d}$, for any $d \geq 1$;

- the skew-shift $(x, y) \mapsto(x+\alpha, y+x)$ on $\mathbb{T}^{2}$, where $\alpha$ is irrational.

1.1. Results for $\operatorname{SL}(2, \mathbb{R})$-Cocycles. Given a continuous map $A: X \rightarrow \operatorname{SL}(2, \mathbb{R})$, we consider the skew-product $X \times \mathrm{SL}(2, \mathbb{R}) \rightarrow X \times \mathrm{SL}(2, \mathbb{R})$ given by $(x, g) \mapsto$ $(f(x), A(x) \cdot g)$. This map is called the cocycle $(f, A)$. For $n \in \mathbb{Z}, A^{n}$ is defined by $(f, A)^{n}=\left(f^{n}, A^{n}\right)$.

We say a cocycle $(f, A)$ is uniformly hyperbolid 1 if there exist constants $c>0, \lambda>1$ such that $\left\|A^{n}(x)\right\|>c \lambda^{n}$ for every $x \in X$ and $n>0$ This is equivalent to the usual hyperbolic splitting condition: see $[\bar{Y}]$. Recall that uniform hyperbolicity is an open condition in $C^{0}(X, \operatorname{SL}(2, \mathbb{R}))$.

We say that two cocycles $(f, A)$ and $(f, \tilde{A})$ are conjugate if there exists a conjugacy $B \in C^{0}(X, \operatorname{SL}(2, \mathbb{R}))$ such that $\tilde{A}(x)=B(f(x)) A(x) B(x)^{-1}$.

Our first result is:

Date: April 22, 2008

This research was partially conducted during the period A. A. served as a Clay Research Fellow. J. B. was partially supported by a grant from CNPq-Brazil. D. D. was supported in part by NSF grant DMS-0653720. We benefited from CNPq and Procad/CAPES support for traveling.

${ }^{1}$ Some authors say that the cocycle has an exponential dichotomy.

${ }^{2}$ If $A$ is a real $2 \times 2$ matrix, then $\|A\|=\sup _{\|v\| \neq 0}\|A(v)\| /\|v\|$, where $\|v\|$ is the Euclidean norm of $v \in \mathbb{R}^{2}$. 
Theorem 1. Let $f$ be as above. If $A: X \rightarrow \operatorname{SL}(2, \mathbb{R})$ is a continuous map such that the cocycle $(f, A)$ is not uniformly hyperbolic, then there exists a continuous $\tilde{A}: X \rightarrow \operatorname{SL}(2, \mathbb{R})$, arbitrarily $C^{0}$-close to $A$, such that the cocycle $(f, \tilde{A})$ is conjugate to an $\mathrm{SO}(2, \mathbb{R})$-valued cocycle.

Remark 1. A cocycle $(f, A)$ is conjugate to a cocycle of rotations if and only if there exists $C>1$ such that $\left\|A^{n}(x)\right\| \leq C$ for every $x \in X$ and $n \in \mathbb{Z}$ (here it is enough to assume that $f$ is minimal); see [Cam, EJ, Y].

Remark 2. In Theorem1, one can drop the hypothesis of unique ergodicity of $f$ (still asking $f$ to be minimal and to fiber over an almost periodic dynamics), as long as $X$ is finite dimensional. See Remark 8

Next we focus on the opposite problem of approximating a cocycle by one that is uniformly hyperbolic. As we will see, this problem is related to the important concept of reducibility.

To define reducibility, we will need a slight variation of the notion of conjugacy. Let us say that two cocycles $(f, A)$ and $(f, \tilde{A})$ are $\operatorname{PSL}(2, \mathbb{R})$-conjugate if there exists $B \in$ $C^{0}\left(X, \operatorname{PSL}(2, \mathbb{R})\right.$ ) such that $\tilde{A}(x)=B(f(x)) A(x) B(x)^{-1}$ (the equality being considered in $\operatorname{PSL}(2, \mathbb{R}))$. We say that $(f, A)$ is reducible if it is $\operatorname{PSL}(2, \mathbb{R})$-conjugate to a constant cocycle.

Remark 3. Reducibility does not imply, in general, that $(f, A)$ is conjugate to a constant cocycle, which would correspond to taking $B \in C^{0}(X, \mathrm{SL}(2, \mathbb{R}))$. For example, let $X=\mathbb{T}^{1}, f(x)=x+\alpha$. Let $H=\operatorname{diag}(2,1 / 2)$, and define $A(x)=R_{-\pi(x+\alpha)} H R_{\pi x}$ Notice $A$ is continuous, $(f, A)$ is $\operatorname{PSL}(2, \mathbb{R})-$, but not $\operatorname{SL}(2, \mathbb{R})-$, conjugate to a constant. (For an example where $(f, A)$ is not uniformly hyperbolic, see Remark 9 )

Let us say that a $\operatorname{SL}(2, \mathbb{R})$-cocycle $(f, A)$ is reducible up to homotopy if there exists a reducible cocycle $(f, \tilde{A})$ such that the maps $A$ and $\tilde{A}: X \rightarrow \operatorname{SL}(2, \mathbb{R})$ are homotopic. Let $R$ uth be the set of all $A$ such that $(f, A)$ is reducible up to homotopy.

Remark 4 . In the case that $f$ is homotopic to the identity map, it is easy to see that $R$ uth coincides with the set of maps $A: X \rightarrow \mathrm{SL}(2, \mathbb{R})$ that are homotopic to a constant.

It is well known that there exists an obstruction to approximating a cocycle by a uniformly hyperbolic one: a uniformly hyperbolic cocycle is always reducible up to homotopy (see Lemma 4). Our next result shows that, up to this obstruction, uniform hyperbolicity is dense.

Theorem 2. Uniform hyperbolicity is dense in Ruth.

This result is obtained as a consequence of a detailed investigation of the problem of denseness of reducibility:

Theorem 3. Reducibility is dense in Ruth. More precisely:

a) If $(f, A)$ is uniformly hyperbolic, then it can be approximated by a reducible cocycle (which is uniformly hyperbolic).

b) If $A \in$ Ruth, but $(f, A)$ is not uniformly hyperbolic and $A_{*} \in \operatorname{SL}(2, \mathbb{R})$ is nonhyperbolic (i.e., $\left.\left|\operatorname{tr} A_{*}\right| \leq 2\right)$, then $(f, A)$ lies in the closure of the PSL $(2, \mathbb{R})$ conjugacy class of $\left(f, A_{*}\right)$.

\footnotetext{
${ }^{3} R_{\theta}$ indicates the rotation of angle $\theta$.
} 
Proof of Theorem 2 The closure of the set of uniformly hyperbolic cocycles is obviously invariant under $\operatorname{PSL}(2, \mathbb{R})$ conjugacies, and clearly contains all constant cocycles $\left(f, A_{*}\right)$ with $\operatorname{tr} A_{*}=2$. The result follows from the second part of Theorem 3 .

Remark 5. It would be interesting to investigate also the closure of an arbitrary $\operatorname{PSL}(2, \mathbb{R})$ conjugacy class. Even the case of the $\operatorname{PSL}(2, \mathbb{R})$ conjugacy class of a constant hyperbolic cocycle already escapes our methods.

Let us say a few words about the proofs and relation with the literature. In the diffeomorphism and flow settings, Smale conjectured in the 1960's that hyperbolic dynamical systems are dense. This turned out to be false in general. However, there are situations where denseness of hyperbolicity holds; see, for example, the recent work [KSS] in the context of one-dimensional dynamics.

Cong $[\mathrm{C}]$ proved that uniform hyperbolicity is (open and) dense in the space of $L^{\infty}(X, \operatorname{SL}(2, \mathbb{R}))$-cocycles, for any base dynamics $f$. So our Theorem 2 can be seen as a continuous version of his result. Cong's proof involves a tower argument to perturb the cocycle and produce an invariant section for its action on the circle $\mathbb{P}^{1}$. We develop a somewhat similar technique, replacing $\mathbb{P}^{1}$ with other spaces. Special care is needed in order to ensure that perturbations and sections be continuous.

Another related result was obtained by Fabbri and Johnson who considered continuous-time systems over translation flows on $\mathbb{T}^{d}$ and proved for a generic translation vector that uniform hyperbolicity occurs for an open and dense set of cocycles; see [FJ].

1.2. Results for Schrödinger Cocycles. We say $(f, A)$ is a Schrödinger cocycle when $A$ takes its values in the set

$$
S=\left\{\left(\begin{array}{cc}
t & -1 \\
1 & 0
\end{array}\right) ; t \in \mathbb{R}\right\} \subset \mathrm{SL}(2, \mathbb{R}) .
$$

The matrices $A^{n}$ arising in the iterates of a Schrödinger cocycle are the so-called transfer matrices associated with a discrete one-dimensional Schrödinger operator.

More explicitly, given $V \in C^{0}(X, \mathbb{R})$ (called the potential) and $x \in X$, we consider the operator

$$
\left(H_{x} \psi\right)_{n}=\psi_{n+1}+\psi_{n-1}+V\left(f^{n} x\right) \psi_{n}
$$

in $\ell^{2}(\mathbb{Z})$. Notice that $u$ solves the difference equation

$$
u_{n+1}+u_{n-1}+V\left(f^{n} x\right) u_{n}=E u_{n}
$$

if and only if

$$
\left(\begin{array}{c}
u_{n} \\
u_{n-1}
\end{array}\right)=A_{E, V}^{n}\left(\begin{array}{c}
u_{0} \\
u_{-1}
\end{array}\right),
$$

where $\left(f, A_{E, V}\right)$ is the Schrödinger cocycle with

$$
A_{E, V}(x)=\left(\begin{array}{cc}
E-V(x) & -1 \\
1 & 0
\end{array}\right) .
$$

Properties of the spectrum and the spectral measures of the operator (2) can be studied by looking at the solutions to (3) and hence, by virtue of (4), the one-parameter family of Schrödinger cocycles $\left(f, A_{E, V}\right)$. Using minimality of $f$, it follows quickly by strong operator convergence that the spectrum of $H_{x}$ is independent of $x \in X$ 
and we may therefore denote it by $\Sigma$. It is well known that $\Sigma$ is a perfect set; as a spectrum it is closed and there are no isolated points by ergodicity of $f$ and finite-dimensionality of the solution space of (3) for fixed $E$. Johnson [J] (see also Lenz [L]) showed that $\Sigma$ consists of those energies $E$, for which $\left(f, A_{E, V}\right)$ is not uniformly hyperbolic:

$$
\mathbb{R} \backslash \Sigma=\left\{E \in \mathbb{R} ;\left(f, A_{E, V}\right) \text { is uniformly hyperbolic }\right\} .
$$

Our results have natural versions for Schrödinger cocycles, with the added simplification that all such cocycles are homotopic to a constant. Simple repetition of the proofs leads to difficulties in the construction of perturbations (since there are fewer parameters to vary). We prove instead a general reduction result, which is of independent interest. Recall the definition (1) of the set $S$.

Theorem 4. Let $f: X \rightarrow X$ be a minimal homeomorphism of a compact metric space. Let $P$ be any conjugacy-invariant property of $\operatorname{SL}(2, \mathbb{R})$-valued cocycles over $f$. If $A \in$ $C^{0}(X, S)$ can be approximated by $\operatorname{SL}(2, \mathbb{R})$-valued cocycles with property $P$, then $A$ can be approximated by $S$-valued cocycles with property $P$.

We are even able to treat the case of more regular cocycles.

Theorem 5. Let $1 \leq r \leq \infty$ and let $0 \leq s \leq r$. Let $X$ be a $C^{r}$ compact manifold and let $f: X \rightarrow X$ be a minimal $C^{r}$-diffeomorphism. Let $P$ be any property of $\operatorname{SL}(2, \mathbb{R})$-valued $C^{r}$ cocycles over $f$ which is invariant by $C^{r}$-conjugacy. If $A \in C^{s}(X, S)$ can be $C^{s}$-approximated by $\mathrm{SL}(2, \mathbb{R})$-valued cocycles with property $P$, then $A$ can be $C^{s}$-approximated by S-valued cocycles with property $P$.

Remark 6. Having in mind applications to other types of difference equations, it would be interesting to investigate the validity of the above results for more general classes of sets.

It follows from Theorems 2 and 4 that uniformly hyperbolic Schrödinger cocycles are $C^{0}$-dense. This has the following corollary:

Corollary 6. For a generic $V \in C^{0}(X, \mathbb{R})$, we have that $\mathbb{R} \backslash \Sigma$ is dense. That is, the associated Schrödinger operators have Cantor spectrum.

Proof. For $E \in \mathbb{R}$, consider the set

$$
U H_{E}=\left\{V \in C^{0}(X, \mathbb{R}) ;\left(f, A_{E, V}\right) \text { is uniformly hyperbolic }\right\} .
$$

By Theorems 2 and $4, U H_{E}$ is (open and) dense. Thus, we may choose a countable dense subset $\left\{E_{n}\right\}$ of $\mathbb{R}$ and then use (5) to conclude that for $V \in \bigcap_{n} U H_{E_{n}}$, the set $\mathbb{R} \backslash \Sigma$ is dense.

Let us discuss this result in the two particular cases of interest, translations and skew-shifts on the torus.

If the base dynamics is given by a translation on the torus, that is, for quasiperiodic Schrödinger operators, Cantor spectrum is widely expected to occur generically. Corollary 6 proves this statement in the $C^{0}$-topology. There are other related results that also establish a genericity statement of this kind. Cong and Fabbri $[\mathrm{CF}]$ consider bounded measurable potentials $V$. Fabbri, Johnson, and Pavani studied quasi-periodic Schrödinger operators in the continuum case, that is, acting in $L^{2}(\mathbb{R})$. They prove for generic translation vector that Cantor spectrum 
is $C^{0}$-generic; see $[\mathrm{FJP}]$. More recently, generic Cantor spectrum for almost periodic Schrödinger operators in the continuum was established by Gordon and Jitomirskaya [GJ].

On the other hand, Corollary 6 is rather surprising in the case of the skewshift. Though few results are known, it is often assumed that in many respects the skew-shift behaves similarly to a Bernoulli shift, and Schrödinger operators associated to Bernoulli shifts never have Cantor spectrum. More precisely, the following is expected for Schrödinger operators defined by the skew-shift and a sufficiently regular non-constant potential function $V: \mathbb{T}^{2} \rightarrow \mathbb{R}$; compare [B4, p. 114]. The (top) Lyapunov exponent of $\left(f, A_{E, V}\right)$ is strictly positive for almost every $E \in \mathbb{R}$, the operator $H_{(x, y)}$ has pure point spectrum with exponentially decaying eigenfunctions for almost every $(x, y) \in \mathbb{T}^{2}$, and the spectrum $\Sigma$ is not a Cantor set. Some partial affirmative results concerning the first two statements can be found in [B2, B3, B4, BGS], whereas Corollary [6above shows that the third expected property fails generically in the $C^{0}$ category ${ }^{4}$ It is natural to pose the question of whether our result is an artifact of weak regularity: can the spectrum of a Schrödinger operator associated to the skew-shift with analytic potential ever be a Cantor set?

The following result will follow quickly from the results described above and the standard KAM theorem:

Theorem 7. Assume that $f$ is a Diophantine translation of the d-torus. Then the set of $V \in C^{0}(X, \mathbb{R})$ for which the corresponding Schrödinger operators have some absolutely continuous spectrum is dense.

This should be compared with [AD], which showed that singular spectrum is $\mathrm{C}^{0}$-generic in the more general context of ergodic Schrödinger operators.

\section{Proof of the Results for SL(2, $\mathbb{R})$-Cocycles}

Our goal is to prove Theorems 1 and 3 .

Lemma 1. There are two possibilities about the group $\mathbb{G}$ :

a) (the circle case) either there is an onto continuous homomorphism $s: G \rightarrow \mathbb{T}^{1}$;

b) (the Cantor case) or $\mathrm{G}$ is a Cantor set.

In the second alternative, there exist continuous homomorphisms from $\mathrm{G}$ onto finite cyclic groups of arbitrarily large order.

In the lack of an exact reference, a proof of Lemma 1 is given in Appendix $\mathrm{A}$

We will first work out the arguments for the more difficult circle case. By assumption, $f$ fibers over a translation on $G$, and hence also over a translation of the circle. That translation is minimal, because so is $f$. Therefore in the circle case, we can and we do assume that $\mathrm{G}=\mathbb{T}^{1}$.

The proofs then go as follows: In \$2.1 we explain a construction of almostinvariant sections for skew-products. This is used in \$2.2 to prove Lemma 3 , which says that functions that are cohomologous to constant are dense in $C^{0}(X, \mathbb{R})$. Using Lemma 3, the first case of Theorem 3 is easily proven in $\$ 2.3$. In $\$ 2.4$ we establish some lemmas about the action of $\operatorname{SL}(2, \mathbb{R})$ on hyperbolic space. The proof of Theorem 1 is given in $\$ 2.5$ It is similar to the proof of Lemma 3 with additional

\footnotetext{
${ }^{4} \mathrm{~A}$ result of a similar flavor was recently obtained in $[\mathrm{BD}]$; if $\alpha$ is not badly approximable, then the second expected property also fails generically in the $C^{0}$ category.
} 
ingredients: results on Lyapunov exponents from $[\mathrm{B} 1]$ and $[\overline{\mathrm{F}}]$ and the material from \$2.4. To prove the second case of Theorem 3 in $\$ 2.6$ we employ Theorem 1 and Lemma 3 again.

In $\$ 2.7$ we will discuss the Cantor case, which is obtained by a simplification of the arguments (because then no gluing considerations are needed).

2.1. Almost-Invariant Sections. From here until \$2.6 we consider only the circle case.

A continuous skew-product over $f$ is a continuous map $F: X \times Y \rightarrow X \times Y$ (where $Y$ is some topological space) of the form $F(x, y)=\left(f(x), F_{x}(y)\right)$. $F$ is called invertible if it is a homeomorphism of $X \times Y$. In this case, we write $F^{n}(x, y)=\left(f^{n}(x), F_{x}^{n}(y)\right)$, for $n \in \mathbb{Z}$. An invariant section for $F$ is a continuous map $x \mapsto y(x)$ whose graph is F-invariant.

Let $p_{i} / q_{i}$ be the $i$ th continued fraction approximation of $\alpha$. We recall that $q_{i} \alpha$ is closer to 0 than any $n \alpha$ with $1 \leq n<q_{i}$; moreover the points $q_{i} \alpha$ alternate sides around 0 .

Let $I_{i} \subset \mathbb{T}^{1}$ be the shortest closed interval containing 0 and $q_{i} \alpha$. Notice that the first $n>0$ for which $I_{i}+n \alpha$ intersects $I_{i}$ is $n=q_{i+1}$. Moreover, $\left(I_{i}+n \alpha\right) \backslash I_{i}$ coincides (modulo a point) with $I_{i+1}$. Also notice that $I_{i+1}+q_{i} \alpha$ is contained in $I_{i}$.

Let $i$ be fixed. The above remarks show that the family of intervals

$$
I_{i}, I_{i}+\alpha, \ldots, I_{i}+\left(q_{i+1}-1\right) \alpha, I_{i+1}, I_{i+1}+\alpha, \ldots, I_{i+1}+\left(q_{i}-1\right) \alpha
$$

has the following properties:

- the union of the intervals is the whole circle;

- the interiors of the intervals are two-by-two disjoint.

(Another way to the obtain the family (6) is to cut the circle along the points $n \alpha$ with $0 \leq n \leq q_{i+1}+q_{i}-1$.) We draw the intervals from (6) from bottom to top as in Figure 1 Then each point is mapped by the $\alpha$-rotation to the point directly above it, or else to somewhere in the bottom floor $I_{i}$.

The following lemma (and its proof) will be used in several situations (namely, $\S \$ 2.2$ and 2.5).

Lemma 2. Let $F: X \times Y \rightarrow X \times Y$ be a continuous invertible skew-product over $f$. Fix any $i \in \mathbb{N}$, and let $I=I_{i}$. Given any map $y_{0}: h^{-1}(I) \rightarrow Y$, there exists a unique map $y_{1}: X \rightarrow Y$ that extends $y_{0}$ and such that

$$
F\left(x, y_{1}(x)\right)=\left(f(x), y_{1}(f(x))\right) \text { for all } x \in X \backslash h^{-1}(I) .
$$

If, in addition, $y_{0}$ is continuous and satisfies

$$
F^{n}\left(x, y_{0}(x)\right)=\left(f^{n}(x), y_{0}\left(f^{n}(x)\right)\right) \text { for all } x \in h^{-1}(0), n \in\left\{q_{i}, q_{i+1}+q_{i}\right\},
$$

then $y_{1}$ is continuous.

Proof. For each $x \in X$, let

$$
\tau(x)=\min \left\{n \geq 0 ; f^{n}(x) \in h^{-1}(I)\right\} .
$$

Given $y_{0}: h^{-1}(I) \rightarrow Y$, then $y_{1}$ must be given by

$$
y_{1}(x)=\left(F_{x}^{\tau(x)}\right)^{-1}\left(y_{0}\left(f^{\tau(x)}(x)\right)\right) .
$$

Now assume that $y_{0}$ is continuous and (8) holds. We only need to check that $y$ is continuous at each point $x$ where $\tau$ is not. Fix such an $x$ and let $k=\tau(x)$. Then either (i) $f^{k}(x) \in h^{-1}(0)$ or (ii) $f^{k}(x) \in h^{-1}\left(q_{i} \alpha\right)$. Let $\ell=\tau\left(f^{k}(x)\right)$, that is, $\ell=q_{i}$ in case (i), and 


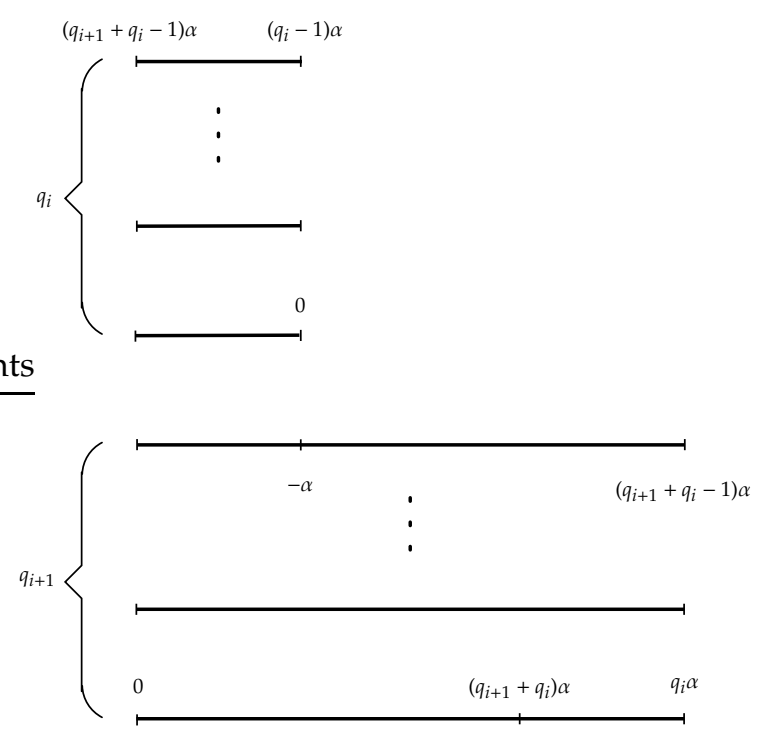

FIgURE 1. Castle with base $I_{i}$.

$\ell=q_{i+1}$ in case (ii). Due to the definition of $y_{0}$, we have $F_{f^{k}(x)}^{\ell}\left(y_{0}\left(f^{k}(x)\right)\right)=y_{0}\left(f^{k+\ell}(x)\right)$ in both cases. Therefore

$$
\left(F_{x}^{k}\right)^{-1}\left(y_{0}\left(f^{k}(x)\right)\right)=\left(F_{x}^{k+\ell}\right)^{-1}\left(y_{0}\left(f^{k+\ell}(x)\right)\right) .
$$

The set of the possible limits of $\tau\left(x_{j}\right)$, where $x_{j} \rightarrow x$, is precisely $\{k, k+\ell\}$. It follows that $y$ is continuous at $x$.

\subsection{The Cohomological Equation.}

Lemma 3. For every $\phi \in C^{0}(X, \mathbb{R})$ and every $\delta>0$, there exists $\tilde{\phi} \in C^{0}(X, \mathbb{R})$ such that $\|\phi-\tilde{\phi}\|_{C^{0}}<\delta$ and $\tilde{\phi}$ is $C^{0}$ cohomologous to a constant: there exist $w \in C^{0}(X, \mathbb{R})$ and $a_{0} \in \mathbb{R}$ such that

$$
\tilde{\phi}=w \circ f-w+a_{0} .
$$

Remark 7. In the case $X=\mathbb{T}^{1}$, there is a quick proof of Lemma 3 Approximate $\phi$ by a (real) trigonometric polynomial $\tilde{\phi}(z)=\sum_{|n| \leq m} a_{n} z^{n}$, and let $w(z)=\sum_{0<|n| \leq m}\left(e^{2 \pi i n \alpha}-\right.$ $1)^{-1} a_{n} z^{n}$

The following proof contains a construction that will appear again in the (harder) proof of Theorem 1, so it may also be useful as a warm-up.

Proof of Lemma 3 Fix $\phi \in C^{0}(X, \mathbb{R})$ and $\delta>0$ small. Let $a_{0}$ be the integral of $\phi$ with respect to the unique $f$-invariant probability measure. Without loss of generality, assume $a_{0}=0$. Write $S_{n}=\sum_{j=0}^{n-1} \phi \circ f^{j}$. Let $n_{0}$ be such that $\left|S_{n} / n\right|<\delta$ uniformly for every $n \geq n_{0}$.

Choose and fix $i$ such that

$$
q_{i}>\max \left(n_{0}, \delta^{-1}\|\phi\|_{C^{0}}\right) .
$$

Let $I=I_{i}$. The rest of the proof is divided into three steps: 
Step 1: Finding an almost-invariant section $w_{1}: X \rightarrow \mathbb{R}$. First define a real function $w_{0}$ on $h^{-1}\left(\left\{0, q_{i} \alpha,\left(q_{i+1}+q_{i}\right) \alpha\right\}\right)$ by

$$
w_{0}\left(f^{n}(x)\right)=S_{n}(x) \text { for } x \in h^{-1}(0) \text { and } n=0, q_{i} \text {, or } q_{i+1}+q_{i} .
$$

Using Tietze's Extension Theorem, we extend continuously $w_{0}$ to $h^{-1}(I)$ so that

$$
\sup _{h^{-1}(I)}\left|w_{0}\right|=\sup _{h^{-1}\left(\left\{0, q_{i} \alpha,\left(q_{i+1}+q_{i}\right) \alpha\right\}\right)}\left|w_{0}\right| .
$$

Now we consider the skew-product

$$
F: X \times \mathbb{R} \rightarrow X \times \mathbb{R}, F(x, w)=(f(x), w+\phi(x)) .
$$

Applying Lemma 2 to $F$ and $w_{0}$, we find a continuous function $w_{1}: X \rightarrow \mathbb{R}$ which extends $w_{0}$ and such that $w_{1}(f(x))=w_{1}(x)+\phi(x)$ if $x \notin h^{-1}$ (int $\left.I\right)$.

Step 2: Definition of functions $\tilde{\phi}, w: X \rightarrow \mathbb{R}$. Define $\tilde{\phi}$ by $\tilde{\phi}=\phi$ outside of $\bigsqcup_{n=0}^{q_{i+1}-1} f^{n}\left(h^{-1}(I)\right)$, and

$$
\tilde{\phi}\left(f^{n}(x)\right)=\phi\left(f^{n}(x)\right)+\frac{w_{1}\left(f^{q_{i+1}}(x)\right)-w_{1}(x)-S_{q_{i+1}}(x)}{q_{i+1}} \quad \text { if } x \in h^{-1}(I), 0 \leq n<q_{i+1} .
$$

Define $w$ by $w=w_{1}$ outside of $\bigsqcup_{n=1}^{q_{i+1}-1} f^{n}\left(h^{-1}(I)\right)$, and

$$
w\left(f^{n}(x)\right)=w(x)+\sum_{j=0}^{n-1} \tilde{\phi}\left(f^{j}(x)\right) \quad \text { if } x \in h^{-1}(I), 0 \leq n<q_{i+1} .
$$

Then $\tilde{\phi}$ and $w$ are continuous functions satisfying $\tilde{\phi}=w \circ f-w$.

Step 3: Distance estimate. From now on, let $x \in h^{-1}(I)$ be fixed. Due to the definition of $w_{0}$ we have

$$
\left|w_{0}(x)\right| \leq\left(q_{i+1}+q_{i}\right) \delta
$$

Recalling (9), we see that $\tau\left(f^{q_{i+1}}(x)\right)$ equals either 1 or $q_{i}+1$ (see Figure1). In any case, $\left|S_{\tau(x)}(x)\right| \leq\left(q_{i}+1\right) \delta$ and therefore, by (10),

$$
\left|w_{1}\left(f^{q_{i+1}}(x)\right)\right| \leq\left|w_{0}\left(f^{\tau(x)+q_{i+1}}(x)\right)\right|+\left|S_{\tau(x)}(x)\right| \leq\left(q_{i+1}+2 q_{i}+1\right) \delta .
$$

Hence

$$
\left|\frac{w_{1}\left(f^{q_{i+1}}(x)\right)-w_{1}(x)}{q_{i+1}}\right| \leq \frac{\left(3 q_{i+1}+3 q_{i}+1\right) \delta}{q_{i+1}}<7 \delta .
$$

That is, the $C^{0}$ distance between $\tilde{\phi}$ and $\phi$ is $<7 \delta$.

2.3. Denseness of Reducibility in the Uniformly Hyperbolic Case. First, let us note a basic fact:

Lemma 4. For any homeomorphism $f: X \rightarrow X$, if $(f, A)$ is uniformly hyperbolic, then $A \in$ Ruth.

Proof. By uniform hyperbolicity, for each $x \in X$ there exists a splitting $\mathbb{R}^{2}=E^{u}(x) \oplus$ $E^{s}(x)$, which depends continuously on $x$ and is left invariant by the cocycle, that is, $A(x) \cdot E^{u, s}(x)=E^{u, s}(f(x))$.

Let $\left\{e_{1}, e_{2}\right\}$ be the canonical basis of $\mathbb{R}^{2}$. For each $x \in X$, let $e^{u}(x) \in E^{u}(x)$ and $e^{s}(x) \in E^{s}(x)$ be unit vectors so that $\left\{e^{u}(x), e^{s}(x)\right\}$ is a positively oriented basis. Define a matrix $B(x)$ putting $B(x) \cdot e_{1}=c e^{u}(x)$ and $B(x) \cdot e_{2}=c e^{s}(x)$, where $c=$ 
$\left[\sin \varangle\left(e^{u}(x), e^{s}(x)\right)\right]^{-1 / 2}$ is chosen so that $\operatorname{det} B(x)=1$. Then $B(x)$ is uniquely defined as an element of $\operatorname{PSL}(2, \mathbb{R})$, and depends continuously on $x$.

Let $D(x)$ be given by $A(x)=B(f(x)) D(x) B(x)^{-1}$. Then $D(x)$ is a diagonal "matrix". Therefore $D: X \rightarrow \operatorname{PSL}(2, \mathbb{R})$ is homotopic to a constant and $A$ is homotopic to a reducible cocycle.

Proof of the first part of Theorem 3 Let us write, for $t \in \mathbb{R}, D_{t}= \pm\left(\begin{array}{cc}e^{t} & 0 \\ 0 & e^{-t}\end{array}\right) \in \operatorname{PSL}(2, \mathbb{R})$.

By the proof of Lemma 4, there exist $B \in C^{0}(X, \operatorname{PSL}(2, \mathbb{R}))$ and $\phi \in C^{0}(X, \mathbb{R})$ such that $A(x)=B(f(x)) D_{\phi(x)} B(x)^{-1}$. By Lemma3, we can perturb $\phi$ (and hence $A$ ) in the $C^{0}$-topology so that $\phi=w \circ f-w+a_{0}$ for some $w \in C^{0}(X, \mathbb{R})$ and $a_{0} \in \mathbb{R}$. We can assume $a_{0} \neq 0$. Then $\hat{B}(x)=B(x) D_{w(x)}$ is a conjugacy between $A$ and the constant $D_{a_{0}}$.

2.4. Disk Adjustment Lemma. The aim here is to establish Lemma 6 below, that will be used in the proof of Theorem 1. First we need to recall some facts about hyperbolic geometry.

The group $\operatorname{SL}(2, \mathbb{R})$ acts on the upper half-plane $\mathbb{H}=\{w \in \mathbb{C} ; \operatorname{Im} w>0\}$ as follows:

$$
A=\left(\begin{array}{ll}
a & b \\
c & d
\end{array}\right) \in \mathrm{SL}(2, \mathbb{R}) \Rightarrow A \cdot w=\frac{a w+b}{c w+d} .
$$

(In fact, the action factors through $\operatorname{PSL}(2, \mathbb{R})$.) We endow the half-plane with the Riemannian metric (of curvature -1 )

$$
v \in T_{w} \mathbb{H} \Rightarrow\|v\|_{w}=\frac{|v|}{\operatorname{Im} w} .
$$

Then $\operatorname{SL}(2, \mathbb{R})$ acts on $\mathbb{H}$ by isometries.

We fix the following conformal equivalence between $\mathbb{H}$ and the unit disk $\mathbb{D}=$ $\{z \in \mathbb{C} ;|z|<1\}$ :

$$
w=\frac{-i z-i}{z-1} \in \mathbb{H} \leftrightarrow z=\frac{w-i}{w+i} \in \mathbb{D} .
$$

We take on the disk the Riemannian metric that makes the map above an isometry, namely $\|v\|_{z}=2\left(1-|z|^{2}\right)^{-2}|v|$. By conjugating, we get an action of $\operatorname{SL}(2, \mathbb{R})$ on $\mathbb{D}$ by isometries, that we also denote as $(A, z) \mapsto A \cdot z$.

Let $d(\cdot, \cdot)$ denote the distance function induced on $\mathbb{D}$ by the Riemannian metric. We claim that:

$$
\|A\|=e^{d(A \cdot 0,0) / 2} \quad \text { for all } A \in \operatorname{SL}(2, \mathbb{R}) .
$$

Proof. It is sufficient to prove the corresponding fact $\|A\|=e^{d(A \cdot i, i) / 2}$ on the halfplane $\mathbb{H}$. We first check the case where $A$ is a diagonal matrix $H_{\lambda}=\left(\begin{array}{cc}\lambda & 0 \\ 0 & \lambda^{-1}\end{array}\right)$ with $\lambda>1$ :

$$
d(A \cdot i, i)=d\left(\lambda^{2} i, i\right)=\int_{1}^{\lambda^{2}} \frac{d y}{y}=2 \log \lambda=2 \log \|A\| .
$$

Next, if $A$ is a rotation $R_{\theta}$ then its action on $\mathbb{H}$ fixes the point $i$, so the claim also holds. Finally, a general matrix can be written as $A=R_{\beta} H_{\lambda} R_{\alpha}$, so (11) follows.

We now prove two lemmas. 
Lemma 5. There exists a continuous map $\Phi: \mathbb{D} \times \mathbb{D} \rightarrow \operatorname{SL}(2, \mathbb{R})$ such that $\Phi\left(p_{1}, p_{2}\right) \cdot p_{1}=$ $p_{2}$ and $\left\|\Phi\left(p_{1}, p_{2}\right)-\mathrm{Id}\right\| \leq e^{d\left(p_{1}, p_{2}\right) / 2}-1$.

Let us recall a few more facts about the half-plane and disk models, that we will use in the proof of the lemma. An extended circle means either an Euclidean circle or an Euclidean line in the complex plane.

- The geodesics on $\mathbb{H}$ (resp. $\mathbb{D}$ ) are arcs of extended circles that meet orthogonally the real axis $\partial \mathrm{H}$ (resp. the unit circle $\partial \mathrm{D}$ ) at the endpoints (called points at infinity).

- The points lying at a fixed positive distance from a geodesic $\gamma$ form two arcs of extended circles $\gamma_{1}$ and $\gamma_{2}$ that have the same points at infinity as $\gamma$. See Figure 2(left). We say that $\gamma$ and $\gamma_{1}$ are equidistant curves.

- A quadrilateral $p_{1} q_{1} q_{2} p_{2}$ is called a Saccheri quadrilateral with base $q_{1} q_{2}$ and summit $p_{1} p_{2}$ if the angles at vertices $q_{1}$ and $q_{2}$ are right and the sides $p_{1} q_{1}$ and $p_{2} q_{2}$ (called the legs) have the same length. See Figure 2 (right). The fact is that the summit is necessarily longer than the base.
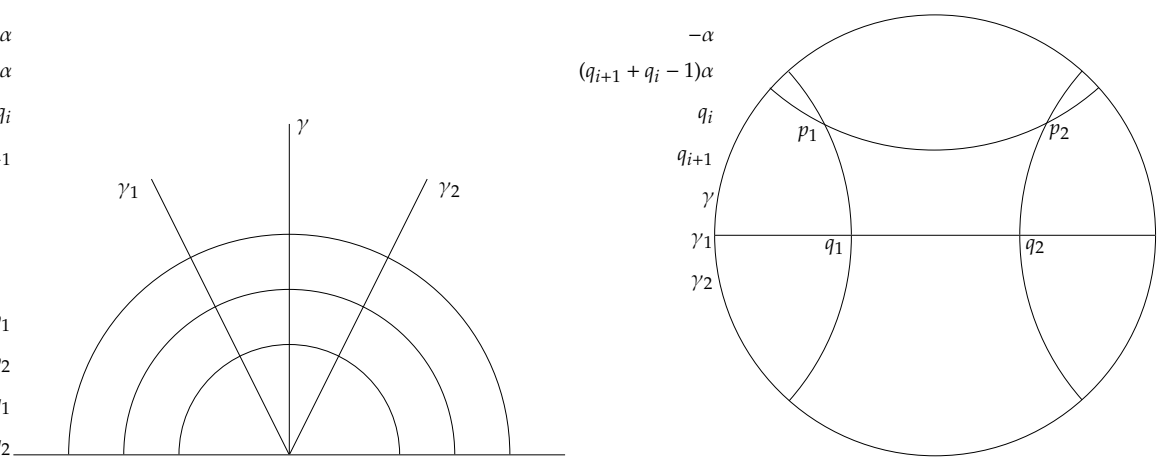

FIguRE 2. Left: equidistant curves on $\mathbb{H}$. Right: a Saccheri quadrilateral $p_{1} q_{1} q_{2} p_{2}$ on $\mathbb{D}$.

Proof of Lemma 5 We will define the matrix $\Phi\left(p_{1}, p_{2}\right)$ explicitly. It is the identity if $p_{1}=p_{2}$, so from now on consider $p_{1} \neq p_{2}$.

We first consider a particular case, where we rewrite the two points as $q_{1}, q_{2}$. Assume that the (whole) geodesic $\gamma$ containing $q_{1}$ and $q_{2}$ also contains 0 . That is, $\gamma$ is a piece of Euclidean line. Let $u$ be the point in the circle $\{|z|=1\}$ such that the line contains $-u, q_{1}, q_{2}, u$, in this order. Consider the hyperbolic isometry that preserves the geodesic $\gamma$, translating it and taking $q_{1}$ to $q_{2}$. That isometry corresponds to a matrix of the form:

$$
A=R_{\theta} H_{\lambda} R_{-\theta}, \quad \text { where } R_{\theta}=\left(\begin{array}{cc}
\cos \theta & -\sin \theta \\
\sin \theta & \cos \theta
\end{array}\right), H_{\lambda}=\left(\begin{array}{cc}
\lambda & 0 \\
0 & \lambda^{-1}
\end{array}\right),
$$

for some $\theta \in \mathbb{R}$ (in fact, $e^{-2 i \theta}=u$ ) and $\lambda>1$. Since the isometry translates $\gamma$, we have $d(0, A \cdot 0)=d\left(q_{1}, A \cdot q_{1}\right)=d\left(q_{1}, q_{2}\right)$. Therefore (11) gives $\lambda=\|A\|=e^{d\left(q_{1}, q_{2}\right) / 2}$. On the other hand, $\|A-\mathrm{Id}\|=\left\|H_{\lambda}-\mathrm{Id}\right\|=\lambda-1$, so we can define $\Phi\left(q_{1}, q_{2}\right)=A$ and the bound claimed in the statement of the lemma becomes an equality.

Next let us consider the general case. Given $p_{1}$ and $p_{2}$, consider the family of extended circles that contain $p_{1}$ and $p_{2}$. There exists a unique $C$ in this family that 


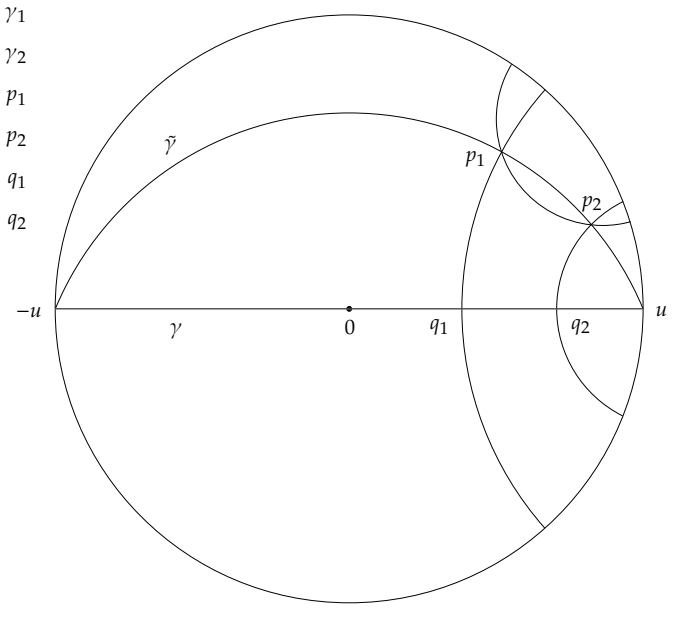

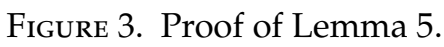

intersects the circle $\{|z|=1\}$ in two antipodal points $u$ and $-u$. (See Figure 3). Let $\tilde{\gamma}=C \cap \mathbb{D}$, and let $\gamma$ be the geodesic whose points at infinity are $u$ and $-u$; so $\gamma$ and $\tilde{\gamma}$ are equidistant curves. Notice that the case already treated corresponds to the case where $\tilde{\gamma}=\gamma$ is a geodesic. Let $q_{1}$, resp. $q_{2}$, be the point in $\gamma$ which has the least hyperbolic distance to $p_{1}$, resp. $p_{2}$. Notice that $\Phi\left(q_{1}, q_{2}\right)$ is already defined. We set $\Phi\left(p_{1}, p_{2}\right)=\Phi\left(q_{1}, q_{2}\right)$.

Because $\gamma$ and $\tilde{\gamma}$ are equidistant, we have $d\left(p_{1}, q_{1}\right)=d\left(p_{2}, q_{2}\right)$. It follows that $p_{1} q_{1} q_{2} p_{2}$ is a Saccheri quadrilateral. In particular, $d\left(q_{1}, q_{2}\right)<d\left(p_{1}, p_{2}\right)$ and hence $\left\|\Phi\left(p_{1}, p_{2}\right)\right\|<e^{d\left(p_{1}, p_{2}\right) / 2}$. Also, since $\Phi\left(q_{1}, q_{2}\right)$ translates the geodesic $\gamma$ sending $q_{1}$ to $q_{2}$, it sends the leg $q_{1} p_{1}$ to the leg $q_{2} p_{2}$, and in particular, sends $p_{1}$ to $p_{2}$, as desired.

This completes the definition of $\Phi$; continuity is evident.

Lemma 6. For every $n \geq 1$, there exists a continuous map $\Psi_{n}: \operatorname{SL}(2, \mathbb{R})^{n} \times \mathbb{D}^{2} \rightarrow$ $\operatorname{SL}(2, \mathbb{R})^{n}$ such that if $\Psi_{n}\left(A_{1}, \ldots, A_{n}, p, q\right)=\left(\tilde{A}_{1}, \ldots, \tilde{A}_{n}\right)$ then

a) $\tilde{A}_{n} \cdots \tilde{A}_{1} \cdot p=q$ and

b) $\left.\left\|\tilde{A}_{i} A_{i}^{-1}-\mathrm{Id}\right\| \leq \exp \left(\frac{1}{2 n} d\left(A_{n} \cdots A_{1} \cdot p, q\right)\right)\right)-1$ for $1 \leq i \leq n$.

Proof. Let $w_{0}=A_{n} \cdots A_{1} \cdot p$ and $L=d\left(w_{0}, q\right)$. For $1 \leq i \leq n$, let $w_{i}$ be the point in the hyperbolic geodesic segment joining $w_{0}$ and $q$ which is at distance $\frac{i L}{n}$ of $w_{0}$. Let also $z_{i}=\left(A_{n} \cdots A_{n-i+1}\right)^{-1} \cdot w_{i}$, for $0 \leq i \leq n$. Then $z_{0}=p, z_{n}=q$ and $d\left(A_{i} \cdot z_{i-1}, z_{i}\right)=\frac{L}{n}$. Let $\tilde{A}_{i}=\Phi\left(A_{i} \cdot z_{i-1}, z_{i}\right) A_{i}$, where $\Phi$ is as in Lemma 5

\subsection{Proof of Theorem 1}

Proof. Let $A: X \rightarrow \mathrm{SL}(2, \mathbb{R})$ be such that $(f, A)$ is not uniformly hyperbolic, and let $\delta_{0}>0$ be given. We want to find $\tilde{A} \in C^{0}(X, \operatorname{SL}(2, \mathbb{R}))$ with $\|\tilde{A}-A\|_{C^{0}}<\delta_{0}$, and a continuous function $z: X \rightarrow \mathbb{D}$ such that $\tilde{A}(x) \cdot z(x)=z(f(x))$. Accomplishing this, we simply set $B(x)=\Phi(z(x), 0)$ (where $\Phi$ is given by Lemma 5 ) and then $B(f(x)) A(x) B(x)^{-1}$ will be rotations.

Because the cocycle is not uniformly hyperbolic, a theorem by Bochi [B1] gives a $C^{0}$-perturbation of $A$ whose upper Lyapunov exponent (with respect to the unique 
invariant probability) is zero. For simplicity of writing, let $A$ denote this perturbation. Since $f$ is uniquely ergodic, a result due to Furman $[\bar{F}]$ gives that $(f, A)$ has uniform subexponential growth, that is,

$$
\lim _{n \rightarrow \infty} \frac{1}{n} \log \left\|A^{n}(x)\right\|=0 \quad \text { uniformly on } x \in X .
$$

Let $\delta>0$ be such that $\left(e^{7 \delta}-1\right)\|A\|_{C^{0}}<\delta_{0}$. Let $n_{0}$ be such that $n \geq n_{0}$ implies $\left\|A^{n}(x)\right\| \leq e^{n \delta}$ for every $x$.

The rest of the argument is analogous to the corresponding steps in the proof of Lemma 3, with the disk playing the role of the line. Let $p_{i} / q_{i}$ be the $i$ th continued fraction approximation of $\alpha$. Choose and fix $i$ large so that

$$
q_{i}>\max \left(n_{0}, \delta^{-1} \log \|A\|_{C^{0}}\right) .
$$

Let $I \subset \mathbb{T}^{1}$ be the shortest closed interval containing 0 and $q_{i} \alpha$. The rest of the proof will be divided into three steps:

Step 1: Finding an almost-invariant section $z_{1}: X \rightarrow \mathbb{R}$. First we define $z_{0}$ on $h^{-1}\left(\left\{0, q_{i} \alpha,\left(q_{i+1}+q_{i}\right) \alpha\right\}\right)$ by

$$
z_{0}\left(f^{n}(x)\right)=A^{n}(x) \cdot 0 \text { for } x \in h^{-1}(0) \text { and } n=0, q_{i} \text {, or } q_{i+1}+q_{i} .
$$

Then we extend continuously $z_{0}$ to $h^{-1}(I)$ in a way such that

$$
\sup _{x \in h^{-1}(I)} d\left(z_{0}(x), 0\right)=\sup _{x \in h^{-1}\left(\left\{0, q_{i} \alpha,\left(q_{i+1}+q_{i}\right) \alpha\right\}\right)} d\left(z_{0}(x), 0\right) .
$$

Consider the skew-product

$$
F: X \times \mathbb{D} \rightarrow X \times \mathbb{D}, F(x, z)=(f(x), A(x) \cdot z) .
$$

Applying Lemma 2 to $F$ and $z_{0}$, we find a continuous map $z_{1}: X \rightarrow \mathbb{D}$ which extends $z_{0}$ and such that $z_{1}(f(x))=A(x) \cdot z_{1}(x)$ if $x \notin h^{-1}$ (int $I$ ).

Step 2: Definition of maps $\tilde{A}: X \rightarrow \operatorname{SL}(2, \mathbb{R})$ and $z: X \rightarrow \mathbb{D}$. Let $\Psi_{q_{i+1}}$ be given by Lemma 6 and put

$$
\begin{aligned}
\left(\tilde{A}(x), \tilde{A}(f(x)), \ldots, \tilde{A}\left(f^{q_{i+1}-1}(x)\right)\right) & = \\
& =\Psi_{q_{i+1}}\left(A(x), A(f(x)), \ldots, A\left(f^{q_{i+1}-1}(x)\right), z_{1}(x), z_{1}\left(f^{q_{i+1}}(x)\right)\right),
\end{aligned}
$$

for each $x \in h^{-1}(I)$. This defines $\tilde{A}$ on $\bigsqcup_{n=0}^{q_{i+1}-1} f^{n}\left(h^{-1}(I)\right)$. Let $\tilde{A}$ equal $A$ on the rest of $X$.

For each $x \in h^{-1}(I)$ and $1 \leq n \leq q_{i+1}-1$, let $z\left(f^{n}(x)\right)=\tilde{A}^{n}(x) \cdot z_{1}(x)$. This defines $z$ on $\bigsqcup_{n=1}^{q_{i+1}-q_{i}-1} f^{n}\left(h^{-1}(I)\right)$. Let $z$ equal $z_{1}$ on the rest of $X$.

It is easy to see that both maps $\tilde{A}$ and $z$ are continuous on the whole $X$, and satisfy $\tilde{A}(x) \cdot z(x)=\tilde{A}(f(x))$.

Step 3: Distance estimate. To complete the proof, we need to check that $\tilde{A}$ is $C^{0}$-close to $A$. Begin noticing that, by relation (11),

$$
B \in \mathrm{SL}(2, \mathbb{R}), w \in \mathbb{D} \Rightarrow d(B \cdot w, 0) \leq d(w, 0)+2 \log \|B\| .
$$

Now fix $x \in h^{-1}(I)$. By the definition of $z_{0}$, we have

$$
d\left(z_{0}(x), 0\right) \leq 2\left(q_{i+i}+q_{i}\right) \delta .
$$


If $y=f^{q_{i+1}}(x)$, then $\tau(y)$ equals 1 or $q_{i}+1$. In either case, $\left\|\left[A^{\tau(y)}(y)\right]^{-1}\right\|=\left\|A^{\tau(y)}(y)\right\| \leq$ $e^{\left(q_{i}+1\right) \delta}$. Since $z_{1}(y)=\left[A^{\tau(y)}(y)\right]^{-1} \cdot z_{0}\left(f^{\tau(y)}(y)\right)$, we get

$$
d\left(z_{1}(y), 0\right) \leq d\left(z_{0}\left(f^{\tau(y)}(y)\right), 0\right)+2 \log \left\|A^{\tau(y)}(y)\right\| \leq 2\left(q_{i+1}+2 q_{i}+1\right) \delta
$$

Putting things together:

$$
\begin{aligned}
d\left(A^{q_{i+1}}(x) \cdot z_{0}(x), z_{1}\left(f^{q_{i+1}}(x)\right)\right) & \leq d\left(A^{q_{i+1}}(x) \cdot z_{0}(x), 0\right)+d\left(0, z_{1}\left(f^{q_{i+1}}(x)\right)\right) \\
& \leq d\left(z_{0}(x), 0\right)+2 \log \left\|A^{q_{i+1}}(x)\right\|+d\left(0, z_{1}\left(f^{q_{i+1}}(x)\right)\right) \\
& \leq 2\left(3 q_{i+1}+3 q_{i}+1\right) \delta .
\end{aligned}
$$

By Lemma 6 ,

$$
\left\|\tilde{A} A^{-1}-\operatorname{Id}\right\|_{C^{0}} \leq \exp \left[\frac{1}{2 q_{i+1}} d\left(A^{q_{i+1}}(x) \cdot z_{0}(x), z_{1}\left(f^{q_{i+1}}(x)\right)\right)\right]-1<e^{7 \delta}-1 .
$$

So $\|\tilde{A}-A\|_{C^{0}} \leq \delta_{0}$, as desired.

Remark 8. A result by Avila and Bochi $[\overline{\mathrm{AB}}]$ says that a generic $\operatorname{SL}(2, \mathbb{R})$-cocycle over a minimal homeomorphism either is uniformly hyperbolic or has uniform subexponential growth (12) (which is equivalent to the simultaneous vanishing of the Lyapunov exponent for all $f$-invariant measures), provided the space $X$ is compact with finite dimension. Using this in the place of the aforementioned results from [B1] and [F], we obtain the generalization claimed in Remark 2 - the rest of the proof is the same.

\subsection{Completion of the Proof of Theorem 3 . We first prove two lemmas:}

Lemma 7. Assume that $A: X \rightarrow \operatorname{SL}(2, \mathbb{R})$ is homotopic to a constant, and that $(f, A)$ is not uniformly hyperbolic. Then there exists $\tilde{A}$ arbitrarily $C^{0}$-close to $A$ and $B \in C^{0}(X, \operatorname{SL}(2, \mathbb{R}))$ such that $B(f(x)) \tilde{A}(x) B(x)^{-1}$ is a constant in $\mathrm{SO}(2, \mathbb{R})$.

Proof. By Theorem 1, we can perturb $A$ so that there exist $A_{1} \in C^{0}(X, \mathrm{SO}(2, \mathbb{R}))$ and $B_{1} \in C^{0}(X, \operatorname{SL}(2, \mathbb{R}))$ such that $A(x)=B_{1}(f(x)) A_{1}(x) B_{1}(x)^{-1}$.

Let $r: \mathrm{SL}(2, \mathbb{R}) \rightarrow \mathrm{SO}(2, \mathbb{R})$ be a deformation retract. Let $B_{2}(x)=r\left(B_{1}(x)\right)$ and $A_{2}(x)=B_{2}(f(x)) A_{1}(x) B_{2}(x)^{-1}$. Then $A_{2}(x)$ is (i) $\mathrm{SO}(2, \mathbb{R})$-valued, (ii) conjugate to $A(x)$, (iii) homotopic to $A(x)$ and therefore to constant.

Due to the existence of the deformation retract $r, A_{2}$ is also homotopic to a constant as a $X \rightarrow \mathrm{SO}(2, \mathbb{R})$ map. Consider the covering map $\mathbb{R} \rightarrow \mathrm{SO}(2, \mathbb{R})$ given by $\theta \mapsto R_{\theta}$. Let $\phi: X \rightarrow \mathbb{R}$ be a lift of $A_{2}$, that is, $A_{2}(x)=R_{\phi(x)}$.

By Lemma 3, there exists $\tilde{\phi}$ very close to $\phi$ such that $\tilde{\phi}=w \circ f-w+a_{0}$ for some $w \in C^{0}(X, \mathbb{R})$ and $a_{0} \in \mathbb{R}$. So the map $\tilde{A}_{2}=R_{\tilde{\phi}(x)}$ is close to $A_{2}$ and conjugate to the constant $R_{a_{0}}$.

Since $A$ and $A_{2}$ are conjugate, there exists $\tilde{A}$ close to $A$ and conjugate to $\tilde{A}_{2}$ (and therefore to the constant). Then $\tilde{A}$ is the map we were looking for.

Lemma 8. If $A \in R$ uth, then $(f, A)$ is $\operatorname{PSL}(2, \mathbb{R})$-conjugate to a cocycle which is homotopic to a constant.

Proof. Let $\pi: \operatorname{SL}(2, \mathbb{R}) \rightarrow \operatorname{PSL}(2, \mathbb{R})$ be the quotient map. Since $A \in R u t h$, there exist $B: X \rightarrow \operatorname{SL}(2, \mathbb{R})$ homotopic to $A, D: X \rightarrow \operatorname{PSL}(2, \mathbb{R})$, and $C \in \operatorname{PSL}(2, \mathbb{R})$ such that $\pi(B(x))=D(f(x)) C D(x)^{-1}$. The map $x \in X \mapsto D(f(x))^{-1} \pi(A(x)) D(x) \in \operatorname{PSL}(2, \mathbb{R})$ is homotopic to a constant; therefore it can be lifted to a map $\tilde{A}: X \rightarrow \operatorname{SL}(2, \mathbb{R})$, which is itself homotopic to a constant. $D$ is a $\operatorname{PSL}(2, \mathbb{R})$-conjugacy between $A$ and $\tilde{A}$. 
Proof of Theorem 3 We have already treated the first part of the theorem, so we will restrict ourselves to the second part.

Fix $A$ and $A_{*}$ as in the statement. By Lemma $8,(f, A)$ is $\operatorname{PSL}(2, \mathbb{R})$-conjugate to a cocycle which is homotopic to a constant. Since the closure of a $\operatorname{PSL}(2, \mathbb{R})$ conjugacy class is invariant under $\operatorname{PSL}(2, \mathbb{R})$ conjugacies, it is enough to consider the case where $A$ is homotopic to a constant. We are going to show that in this case $(f, A)$ lies in the closure of the $\operatorname{SL}(2, \mathbb{R})$-conjugacy class of $\left(f, A_{*}\right)$.

By Lemma 77, $A$ can be perturbed to become conjugate to a constant $C_{*}=R_{\theta}$ in $\mathrm{SO}(2, \mathbb{R})$. We will explain how to perturb $C_{*}$ (and hence $A$, because the conjugacy between $C_{*}$ and $A$ is fixed) in order that $\left(f, C_{*}\right)$ becomes conjugate to $\left(f, A_{*}\right)$. There are two cases, depending on $A_{*}$.

In the case where $A_{*}=\operatorname{Id}$ or $A_{*}$ is elliptic (i.e., $\left.\left|\operatorname{tr} A_{*}\right|<2\right)$, there exist $B_{*} \in \mathrm{SL}(2, \mathbb{R})$ and $\beta \in \mathbb{R}$ such that $A_{*}=B_{*}^{-1} R_{\beta} B_{*}$. Since $\alpha$ is irrational, we can choose $k \in \mathbb{Z}$ such that $2 \pi k \alpha+\theta$ is very close to $\beta$ (modulo $2 \pi \mathbb{Z}$ ). We still have the right to perturb $\theta$, so we can assume $2 \pi k \alpha+\theta=\beta$. Letting $B(x)=B_{*} R_{2 \pi k h(x)}$, we see that $B(f(x)) R_{\theta} B(x)^{-1}$ is precisely $A_{*}$. So $\left(f, R_{\theta}\right)$ is conjugate to $\left(f, A_{*}\right)$, as desired.

In the remaining case, $A_{*}$ is parabolic (i.e., $\operatorname{tr} A_{*}= \pm 2$ ) with $A_{*} \neq \pm \mathrm{Id}$. By the previous case, we can assume $C_{*}= \pm$ Id to start. In fact, we can perturb further and assume $C_{*}$ is a parabolic matrix with $C_{*} \neq \pm \mathrm{Id}$. Then $C_{*}$ and $A_{*}$ are automatically conjugate in the group $\operatorname{SL}(2, \mathbb{R})$, so we are done.

Remark 9. Assuming that $A$ is homotopic to some cocycle which is conjugate to a constant, "PSL $(2, \mathbb{R})$ conjugacy class" can be replaced by "SL $(2, \mathbb{R})$ conjugacy class" in the second part of Theorem 3 . We give an example showing that the stronger conclusion does not hold without additional hypotheses. Let $f: \mathbb{T}^{2} \rightarrow \mathbb{T}^{2}$ be given by $(x, y) \mapsto(x+\alpha, y+2 x)$. Let $A(x, y)=R_{2 \pi x}$. We claim that:

a) $(f, A)$ is reducible.

b) For any $\tilde{A}$ close to $A,(f, \tilde{A})$ is not conjugate to a constant $\operatorname{SL}(2, \mathbb{R})$-cocycle.

To prove (a), let $D(x, y)=R_{\pi y}$ (which is well-defined in $\operatorname{PSL}(2, \mathbb{R})$ ), and notice $D(f(x, y)) D(x, y)^{-1}=A(x, y)$. To prove (b), we will show that for any continuous $B: \mathbb{T}^{2} \rightarrow \mathrm{SL}(2, \mathbb{R})$, the map $C(x, y)=B(f(x, y)) A(x, y) B(x, y)^{-1}$ is not homotopic to a constant. Consider the homology groups $H_{1}\left(\mathbb{T}^{2}\right)=\mathbb{Z}^{2}$ and $H_{1}(\operatorname{SL}(2, \mathbb{R}))=\mathbb{Z}$, and the induced homomorphisms

$$
f_{*}:(m, n) \mapsto(m, 2 m+n), \quad A_{*}:(m, n) \mapsto m, \quad B_{*}:(m, n) \mapsto k m+\ell n .
$$

We have $C_{*}=B_{*} \circ f_{*}+A_{*}-B_{*}$, therefore $C_{*}:(m, n) \mapsto(2 \ell+1) m$ cannot be the zero homomorphism.

2.7. The Case of Cantor Groups. Now assume the second case in Lemma 1, So there are integers $q_{i} \rightarrow \infty$ and continuous homomorphisms $h_{i}: G \rightarrow \mathbb{T}^{1}$ such that the image of $h_{i}$ is the (cyclic) subgroup of $\mathbb{T}^{1}$ of order $q_{i}$. Notice that the level sets of $h_{i}$ are compact, open, and are cyclically permuted by $f$. They form a tower of height $q_{i}$ that will replace the more complicate castle of Figure1 in our arguments. Changing the definition of $h_{i}$, we can assume that $h_{i}(f(x))=h_{i}(x)+\frac{1}{q_{i}}(\bmod 1)$.

There are only three proofs that need modification:

\footnotetext{
${ }^{5}$ Recall that if $G$ is a path-connected topological group and $\gamma_{1}, \gamma_{2}, \gamma:[0,1] \rightarrow G$ are such that $\gamma(t)=\gamma_{1}(t) \gamma_{2}(t)$, then the 1-chains $\gamma$ and $\gamma_{1}+\gamma_{2}$ are homologous.
} 
Proof of Lemma 3 in the Cantor case. Fix $\phi \in C^{0}(X, \mathbb{R})$ with mean zero, and let $\delta>0$ small. Let $n_{0}$ be such that $n \geq n_{0} \Rightarrow\left|S_{n} / n\right|<\delta$ uniformly, where $S_{n}$ is the $n$th Birkhoff sum of $f$. Choose $i$ such that $q_{i}>n_{0}$. Define $\tilde{\phi}$ and $w: X \rightarrow \mathbb{R}$ by

$$
x \in h_{i}^{-1}(0), 0 \leq n<q_{i} \Rightarrow \tilde{\phi}\left(f^{n}(x)\right)=\phi\left(f^{n}(x)\right)-\frac{S_{q_{i}}(x)}{q_{i}}, w\left(f^{n}(x)\right)=S_{n}(x)-\frac{n S_{q_{i}}(x)}{q_{i}} .
$$

Then $\tilde{\phi}$ and $w$ are continuous, $\tilde{\phi}=w \circ f-w$, and $|\tilde{\phi}-\phi|<\delta$.

Proof of Theorem 1 in the Cantor case. Assume $(f, A)$ is not uniformly hyperbolic. Given $\delta_{0}>0$, let $\delta>0$ be such that $\left(e^{\delta}-1\right)\|A\|_{C^{0}}<\delta_{0}$. Perturbing $A$, we can assume $\left\|A^{n}(x)\right\|<e^{n \delta}$ for every $n \geq n_{0}=n_{0}(\delta)$. Fix $q_{i}>n_{0}$. Define $\tilde{A}: X \rightarrow \operatorname{SL}(2, \mathbb{R})$ so that $\left(\tilde{A}(x), \tilde{A}(f(x)), \ldots, \tilde{A}\left(f^{q_{i}-1}(x)\right)\right)=\Psi_{q_{i}}\left(A(x), A(f(x)), \ldots, A\left(f^{q_{i+1}-1}(x)\right), 0,0\right), \forall x \in h_{i}^{-1}(0)$, where $\Psi_{q_{i}}$ is given by Lemma 6 Define $z: X \rightarrow \mathbb{D}$ by $z\left(f^{n}(x)\right)=\tilde{A}^{n}(x) \cdot 0$ for $x \in$ $h_{i}^{-1}(0)$ and $0 \leq n<q_{i}$. Then $\tilde{A}$ and $z$ are continuous, and satisfy $\tilde{A}(x) \cdot z(x)=\tilde{A}(f(x))$. Moreover, since $d\left(A^{q_{i}}(0), 0\right)<q_{i} \delta$, we have $\|\tilde{A}-A\|<\left(e^{\delta}-1\right)\|A\|<\delta_{0}$.

Proof of Theorem 3 (b) in the Cantor case. We only need to show that the closure of the $\mathrm{SO}(2, \mathbb{R})$-conjugacy class of a constant $\mathrm{SO}(2, \mathbb{R})$-valued cocycle contains all constant $\mathrm{SO}(2, \mathbb{R})$-valued cocycles. Given a constant cocycle $R_{\theta}, i \in \mathbb{N}$, and $k \in \mathbb{Z}$, let $B(x)=R_{2 \pi k h_{i}(x)}$. Then $B(f(x)) R_{\theta} B(x)^{-1}=R_{\theta+2 \pi k / q_{i}}$. So the claim follows.

This completes the proofs of Theorems 1 and 3 ,

\section{Proof of the Results for Schrödinger Cocycles}

In this section, we will prove Theorems 4, 5, and 7 .

3.1. Projection Lemma. The proof of Theorems 4 and 5 is based on the following "projection lemma":

Lemma 9. Let $0 \leq r \leq \infty$. Let $f: X \rightarrow X$ be a minimal homeomorphism of a compact metric space with at least three points (if $r=0$ ) or a minimal $C^{r}$ diffeomorphism of a $C^{r}$ compact manifold. Let $A \in C^{r}(X, S)$ be a map whose trace is not identically zero. Then there exist a neighborhood $W \subset C^{0}(X, S L(2, \mathbb{R}))$ of $A$ and continuous maps

$$
\Phi=\Phi_{A}: \mathcal{W} \rightarrow C^{0}(X, S) \text { and } \Psi=\Psi_{A}: \mathcal{W} \rightarrow C^{0}(X, \operatorname{SL}(2, \mathbb{R}))
$$

satisfying:

$$
\begin{gathered}
\Phi \text { and } \Psi \text { restrict to continuous maps } W \cap C^{s} \rightarrow C^{s}, \text { for } 0 \leq s \leq r, \\
\qquad(B)(f(x)) \cdot B(x) \cdot[\Psi(B)(x)]^{-1}=\Phi(B)(x), \\
\Phi(A)=A \text { and } \Psi(A)=\mathrm{id} .
\end{gathered}
$$

Proof of Theorems 4 and 5 The result is easy if $\# X \leq 2$, so we will assume that $\# X \geq 3$. In this case, Lemma 9 implies the result unless $\operatorname{tr} A$ is identically 0.

Assume that $\operatorname{tr} A$ is identically 0 . Let $V \subset X$ be an open set such that $\bar{V} \cap f(\bar{V})=\varnothing$ and $\bar{V} \cap f^{2}(\bar{V})=\varnothing$. Let $\tilde{A} \in C^{r}(X, S)$ be $C^{r}$ close to $A$ such that $\operatorname{tr} \tilde{A}$ is supported in $V \cup f^{2}(V)$ and moreover $\operatorname{tr} \tilde{A}(z)+\operatorname{tr} \tilde{A}\left(f^{2}(z)\right)=0$ for $z \in V$. Then $(f, \tilde{A})$ is $C^{r}$ conjugate to $A$ : Letting $B(x)=$ id for $x \notin f(V) \cup f^{2}(V), B(x)=\tilde{A}\left(f^{-1}(x)\right) R_{-\pi / 2}$ for $x \in f(V)$, and $B(x)=R_{-\pi / 2} \tilde{A}\left(f^{-2}(x)\right)$ for $x \in f^{2}(V)$, we have $B(f(x)) A(x) B(x)^{-1}=\tilde{A}(x)$. If $A$ can be $C^{s}$ approximated by $\mathrm{SL}(2, \mathbb{R})$-valued cocycles with property $P$, then so can $\tilde{A}$. Since 
$\operatorname{tr} \tilde{A}$ does not vanish identically, $\tilde{A}$ can be $C^{s}$-approximated by $S$-valued cocycles with property $P$. Since $\tilde{A}$ can be chosen arbitrarily $C^{r}$ close to $A$, we conclude that $A$ can be $C^{S}$-approximated by $S$-valued cocycles with property $P$.

The proof of Lemma 9 has two distinct steps. First we show that $\operatorname{SL}(2, \mathbb{R})$ perturbations can be conjugated to localized $\operatorname{SL}(2, \mathbb{R})$ perturbations and then we show how to conjugate localized perturbations to Schrödinger perturbations.

In order to be precise, the following definition will be useful. Given $A \in$ $C^{r}(X, \mathrm{SL}(2, \mathbb{R}))$ and $K \subset X$ compact, let $C_{A, K}^{r}(X, \mathrm{SL}(2, \mathbb{R})) \subset C^{r}(X, \mathrm{SL}(2, \mathbb{R}))$ be the set of all $B$ such that $B(x)=A(x)$ for $x \notin K$. The two steps we described correspond to the following two lemmas.

Lemma 10. Let $V \subset X$ be any nonempty open set and let $A \in C^{r}(X, \operatorname{SL}(2, \mathbb{R}))$ be arbitrary. Then there exist an open neighborhood $W_{A, V} \subset C^{0}(X, S L(2, \mathbb{R}))$ of $A$ and continuous maps $\Phi=\Phi_{A, V}: \mathcal{W}_{A, V} \rightarrow C_{A, \bar{V}}^{0}(X, \operatorname{SL}(2, \mathbb{R}))$ and $\Psi=\Psi_{A, V}: \mathcal{W}_{A, V} \rightarrow C^{0}(X, \operatorname{SL}(2, \mathbb{R}))$ satisfying (13), (14), and (15).

Lemma 11. Let $K \subset X$ be a compact set such that $K \cap f(K)=\varnothing$ and $K \cap f^{2}(K)=\varnothing$. Let $A \in C^{r}(X, S)$ be such that for every $z \in K$ we have $\operatorname{tr} A(z) \neq 0$. Then there exists an open neighborhood $W_{A, K} \subset C_{A, K}^{0}(X, \operatorname{SL}(2, \mathbb{R}))$ of $A$ and continuous maps

$$
\Phi=\Phi_{A, K}: \mathcal{W}_{A, K} \rightarrow C^{0}(X, S) \text { and } \Psi=\Psi_{A, K}: \mathcal{W}_{A, K} \rightarrow C^{0}(X, \operatorname{SL}(2, \mathbb{R}))
$$

satisfying (13), (14), and (15).

Before proving the two lemmas, let us show how they imply Lemma 9.

Proof of Lemma 9 Let $z \in X$ be such that $\operatorname{tr} A(z) \neq 0$. Let $V$ be an open neighborhood of $z$ such that with $K=\bar{V}$ we have $\operatorname{tr} A(x) \neq 0$ for $x \in K, K \cap f(K)=\varnothing$, and $K \cap f^{2}(K)=$ $\varnothing$. Let $\Phi_{A, V}: \mathcal{W}_{A, V} \rightarrow C_{A, K}^{0}(X, \operatorname{SL}(2, \mathbb{R}))$ and $\Psi_{A, V}: \mathcal{W}_{A, V} \rightarrow C^{0}(X, \operatorname{SL}(2, \mathbb{R}))$ be given by Lemma10 Let $\Phi_{A, K}: \mathcal{W}_{A, K} \rightarrow C^{0}(X, S)$ and $\Psi_{A, K}: \mathcal{W}_{A, K} \rightarrow C^{0}(X, \operatorname{SL}(2, \mathbb{R}))$ be given by Lemma 11. Let $\mathcal{W}$ be the domain of $\Phi=\Phi_{A, K} \circ \Phi_{A, V}$ and let $\Psi=$ $\left(\Psi_{A, K} \circ \Phi_{A, V}\right) \cdot \Psi_{A, V}$. The result follows.

Proof of Lemma 10 For every $x \in X$, let $y=y_{x} \in V$ and $n=n_{x} \geq 0$ be such that $f^{n}(y)=x$, but $f^{-i}(x) \notin V$ for $0 \leq i \leq n-1$. Let $W=W_{x} \subset V$ be an open neighborhood of $y$ such that $W \cap f^{i}(W)=\varnothing$ for $1 \leq i \leq n$. Let $K=K_{x} \subset W$ be a compact neighborhood of $y$. Let $U=U_{x} \subset f^{n}\left(K_{x}\right)$ be an open neighborhood of $x$. Let $\phi=\phi_{x}: W \rightarrow[0,1]$ be a $C^{r}$ map such that $\phi(z)=0$ for $z \in W \backslash K$, while $\phi(z)=1$ for $z \in f^{-n}(U)$.

Define maps $\Phi_{x}, \Psi_{x}: \mathcal{W}_{x} \rightarrow C^{0}(X, \operatorname{SL}(2, \mathbb{R}))$ on some open neighborhood $\mathcal{W}_{x}$ of $A$ as follows. Let $\Pi: \mathrm{GL}^{+}(2, \mathbb{R}) \rightarrow \mathrm{SL}(2, \mathbb{R})$ be given by $\Pi(M)=(\operatorname{det} M)^{-1 / 2} M$. Let $\Phi_{x}(B)(z)=B(z)$ for $z \notin \bigcup_{i=0}^{n} f^{i}(W), \Phi_{x}(B)(z)=\Pi\left(B(z)+\phi\left(f^{-j}(z)\right)(A(z)-B(z))\right)$ for $z \in f^{j}(W)$ and $1 \leq j \leq n$, and

$$
\Phi_{x}(B)(z)=\left[\Phi_{x}(B)(f(z))\right]^{-1} \cdots\left[\Phi_{x}(B)\left(f^{n}(z)\right)\right]^{-1} \cdot B\left(f^{n}(z)\right) \cdots B(z) \text { for } z \in W .
$$

Let $\Psi_{x}(B)(z)=$ id for $x \notin \bigcup_{i=1}^{n} f^{i}(W)$ and $\Psi_{x}(B)(z)=\Phi_{x}(B)\left(f^{-1}(z)\right) \cdots \Phi_{x}(B)\left(f^{-j}(z)\right)$. $B\left(f^{-j}(z)\right)^{-1} \cdots B\left(f^{-1}(z)\right)^{-1}$ for $z \in f^{j}(W)$ and $1 \leq j \leq n$. Then $\Phi_{x}$ and $\Psi_{x}$ are continuous and have the following properties:

a) For every $z \in X$, we have $\Psi_{x}(B)(f(z)) \cdot B(z) \cdot\left[\Psi_{x}(B)(z)\right]^{-1}=\Phi_{x}(z)$;

b) The set $\left\{z \in X \backslash V ; \Phi_{x}(B)(z)=A(z)\right\}$ contains $\{z \in X \backslash V ; B(z)=A(z)\} \cup\left(U_{x} \backslash V\right)$; 
c) $\Phi_{x}(A)=A$ and $\Psi_{x}(A)=$ id.

Choose a finite sequence $x_{1}, \ldots, x_{k}$ such that $X=\bigcup_{i=1}^{k} U_{x_{i}}$. Let $\mathcal{W}_{A, V} \subset C^{0}(X, \operatorname{SL}(2, \mathbb{R}))$ be an open neighborhood of $A$ such that $\Phi_{i}=\Phi_{x_{i}} \circ \cdots \circ \Phi_{x_{1}}$ is well defined for $1 \leq i \leq k$. Let $\Psi_{i}=\Psi_{x_{i}} \circ \Phi_{i-1}$ for $2 \leq i \leq k$ and $\Psi_{1}=\Psi_{x_{1}}$. The result follows with $\Phi=\Phi_{k}$ and $\Psi=\Psi_{k} \cdots \Psi_{1}$.

Proof of Lemma 11 Let $Z \subset S^{3}$ be the set of all $\left(B_{1}, B_{2}, B_{3}\right)$ such that $\operatorname{tr} B_{2} \neq 0$. One easily checks that $\left(B_{1}, B_{2}, B_{3}\right) \mapsto B_{3} B_{2} B_{1}$ is an analytic diffeomorphism between $Z$ and

$$
L=\left\{\left(\begin{array}{ll}
a & b \\
c & d
\end{array}\right) \in \operatorname{SL}(2, \mathbb{R}) ; d \neq 0\right\} .
$$

Let $\eta: L \rightarrow Z$ be the inverse map.

Let $\Phi(B)(x)=A(x)$ if $z \notin \bigcup_{i=-1}^{1} f^{i}(K)$ and for $z \in K$, let

$$
\left(\Phi(B)\left(f^{-1}(z)\right), \Phi(B)(z), \Phi(B)(f(z))\right)=\eta\left(B\left(f^{-1}(z)\right), B(z), B(f(z))\right) .
$$

Let $\Psi(B)(z)=$ id for $z \notin K \cup f(K), \Psi(z)=\Phi(B)\left(f^{-1}(z)\right) \cdot\left[B\left(f^{-1}(z)\right)\right]^{-1}$ for $z \in K$ and $\Psi(z)=\Phi(B)\left(f^{-1}(z)\right) \cdot \Phi(B)\left(f^{-2}(z)\right) \cdot\left[B\left(f^{-2}(z)\right)\right]^{-1} \cdot\left[B\left(f^{-1}(z)\right)\right]^{-1}$ for $z \in f(K)$. All properties are easy to check.

Remark 10. Let $f: X \rightarrow X$ be a homeomorphism of a compact metric space and let $N \geq n \geq 1$. Let us say that a compact set $K$ is $(n, N)$-good if $K \cap f^{k}(K)=\varnothing$ for $1 \leq k \leq n-1$ and $\bigcup_{k=0}^{N-1} f^{k}(K)=X$. Then Lemma 9 holds under the weaker (than minimality of $f$ ) hypothesis that there exist $N \geq 3$ and a $(3, N)$-good compact set $K$ such that $\operatorname{tr} A(x) \neq 0$ for every $x \in K$.

3.2. Dense Absolutely Continuous Spectrum. To prove Theorem 7 , we will use the following standard result:

Theorem 8. Let $f$ be a Diophantine translation of the d-torus $\mathbb{T}^{d}=\mathbb{R}^{d} / \mathbb{Z}^{d}$. Then there exists a set $\Theta \subset \mathbb{R}$ of full Lebesgue measure such that if $V \in C^{\infty}\left(\mathbb{T}^{d}, \mathbb{R}\right)$ and $E_{0} \in \mathbb{R}$ are such that $\left(f, A_{E_{0}, V}\right)$ is $C^{\infty} \operatorname{PSL}(2, \mathbb{R})$ conjugate to $\left(f, R_{\pi \theta}\right)$ for some $\theta \in \Theta$, then the associated Schrödinger operator has some absolutely continuous spectrum.

For completeness, we will discuss the reduction of this result to the standard KAM Theorem in more detail in Appendix B

Proof of Theorem $\nabla$ Let $V \in C^{0}\left(\mathbb{T}^{d}, \mathbb{R}\right)$ be non-constant, and let $E$ be in the spectrum of the associated Schrödinger operator. By Lemma 7 there exists $\tilde{A} \in$ $C^{0}\left(\mathbb{T}^{d}, \mathrm{SL}(2, \mathbb{R})\right)$ arbitrarily close to $A_{E, V}$ such that $(f, \tilde{A})$ is conjugate to $\left(f, R_{2 \pi \theta}\right)$ for some $\theta \in \Theta$. Approximating the conjugacy by a $C^{\infty}$ map, we may assume that $\tilde{A}$ is $C^{\infty}$. Applying Lemma 9 with $r=\infty$, we find a $C^{\infty}$ function $\tilde{V}$ which is $C^{0}$-close to $V$, such that $\left(f, A_{E, \tilde{V}}\right)$ is conjugate to $(f, A)$, and hence to $\left(f, R_{2 \pi \theta}\right)$. The result now follows from Theorem 8 .

\section{Appendix A. Topological Groups}

We quickly review some material that can be found in $[\mathbb{R}]$. Let $G$ be a topological group. If $G$ is locally compact and abelian, one defines the dual group $\Gamma=\hat{G}$; it consists of all characters of $G$ (i.e., continuous homomorphisms $\gamma: G \rightarrow \mathbb{T}^{1}$ ). Then $\Gamma$ is an abelian group, and with the suitable topology, it is also locally compact. 
Some important facts are: (1) $G$ is compact iff $\Gamma$ is discrete; (2) Pontryagin Duality6: $\hat{\Gamma}=G$.

Proof of Lemma 1 Since $\mathrm{G}$ is compact and infinite, the dual group $\Gamma$ is discrete and infinite.

First, assume that $\Gamma$ contains an element of infinite order. So we can assume $\mathbb{Z}$ is a closed subgroup of $\Gamma$. Let $\iota: \mathbb{Z} \rightarrow \Gamma$ be the inclusion homomorphism, and let $s: \hat{\Gamma} \rightarrow \hat{\mathbb{Z}}$ be its dual . Then $s$ is onto: every character on $\mathbb{Z}$ can be extended to a character on $\Gamma$; see $\left[\mathbb{R}\right.$, §2.1.4]. Since $\hat{\Gamma}=\mathbb{G}$ and $\hat{\mathbb{Z}}=\mathbb{T}^{1}$, alternative (a) holds.

Now, assume that all elements of $\Gamma$ are of finite order. Then $\mathbb{G}$ is a Cantor set, see [R, §2.5.6]. There is a translation $x \mapsto x+\alpha$ of $G$ which is a factor of the minimal homeomorphism $f$, and so it is itself minimal. Therefore $\Gamma$ is a subgroup of $\mathbb{T}_{d^{\prime}}^{1}$ the circle group with the discrete topology; see [R, §2.3.3]. So there exist cyclic subgroups $\Lambda_{i} \subset \Gamma$ with $\left|\Lambda_{i}\right| \rightarrow \infty$. Let $H_{i} \subset \mathbb{G}$ be the annihilator (see [R, §2.1]) of $\Lambda_{i}$. Then $\mathbb{G} / H_{i}=\Lambda_{i}$, and the quotient homomorphism $\mathbb{G} \rightarrow \mathbb{G} / H_{i}$ is continuous. So we are in case (b).

\section{Appendix B. Absolutely Continuous Spectrum via KAM}

In this section, $f: \mathbb{T}^{d} \rightarrow \mathbb{T}^{d}$ is a minimal translation of the torus $\mathbb{T}^{d}=\mathbb{R}^{d} / \mathbb{Z}^{d}$. We will show how Theorem 8 reduces to a result of $[\mathrm{H}]$, based on the usual KAM theorem. We will use the following criterion for absolutely continuous spectrum (see, e.g., [S] for a simple proof):

Lemma 12. Let $f: \mathbb{T}^{d} \rightarrow \mathbb{T}^{d}$ be a homeomorphism, and let $V \in C^{0}\left(\mathbb{T}^{d}, \mathbb{R}\right)$. If $\Sigma_{b}=\{E \in$ $\mathbb{R},\left(f, A_{E, V}\right)$ is conjugate to a cocycle of rotations $\}$ has positive Lebesgue measure, then the associated Schrödinger operators have some absolutely continuous spectrum.

Recall that if $A: \mathbb{T}^{d} \rightarrow \mathrm{SL}(2, \mathbb{R})$ is homotopic to a constant, one can define a fibered rotation number $\rho(f, A) \in \mathbb{R} / \mathbb{Z}$ (see $[\mathrm{H}]$ ). The following properties of the fibered rotation number are easy to check:

a) If $A$ is a constant rotation of angle $\pi \theta$, then $\rho(f, A)=\theta$.

b) If $B$ is a $\operatorname{PSL}(2, \mathbb{R})$ conjugacy between $(f, A)$ and $\left(f, A^{\prime}\right)$, then $\rho(f, A)=$ $\rho\left(f, A^{\prime}\right)+k \alpha$, where $k=k(B) \in \mathbb{Z}$ only depends on the homotopy class of $B$.

c) The fibered rotation number is a continuous function of $A \in C^{0}\left(\mathbb{T}^{d}, \operatorname{SL}(2, \mathbb{R})\right)$.

d) If $A \in C^{0}\left(\mathbb{T}^{d}, \mathrm{SL}(2, \mathbb{R})\right)$ is $\operatorname{PSL}(2, \mathbb{R})$ conjugate to a constant rotation, then the fibered rotation number (as a function of $C^{0}\left(\mathbb{T}^{d}, \mathrm{SL}(2, \mathbb{R})\right.$ ) is $K$-Lipschitz at $A$ for some $K>08$

In $[\overline{\mathrm{H}}]$, it is shown how the KAM Theorem implies reducibility for cocycles close to constant, under a Diophantine assumption on $f$ and on the fibered rotation number. To state it precisely, it will be convenient to introduce the following notation.

Let $n \geq 1$ and $\kappa, \tau>0$. Let $\mathrm{DC}_{n, \kappa, \tau}$ be the set of all $\alpha \in \mathbb{R}^{n}$ such that there exists $\kappa, \tau>0$ such that for every $k_{0} \in \mathbb{Z}, k \in \mathbb{Z}^{n} \backslash\{0\}$, we have $\left|k_{0}+\sum_{i=1}^{n} k_{i} \alpha_{i}\right|>$

\footnotetext{
$6 “="$ means isomorphic and homeomorphic.

${ }^{7}$ The dual of a continuous homomorphism $h: G_{1} \rightarrow G_{2}$ is the continuous homomorphism $\hat{h}: \hat{G}_{2} \rightarrow \hat{G}_{1}$ defined by $\left\langle x_{1}, \hat{h}\left(\gamma_{2}\right)\right\rangle=\left\langle h\left(x_{1}\right), \gamma_{2}\right\rangle$, where $x_{1} \in G_{1}, \gamma_{2} \in \hat{G}_{2}$.

${ }^{8}$ Let $\phi: Y \rightarrow Z$ be a function between metric spaces and let $K>0$. We say that $\phi$ is $K$-Lipschitz at $y \in Y$ if there exists a neighborhood $\mathcal{V} \subset Y$ of $y$ such that for every $z \in \mathcal{V}, d_{Z}(\phi(z), \phi(y)) \leq K d_{Y}(z, y)$.
} 
$\kappa\left(\sum_{i=1}^{n}\left|k_{i}\right|\right)^{-\tau}$. Notice that $\mathrm{DC}_{n, \kappa, \tau}$ is $\mathbb{Z}^{d}$ invariant. Let $\mathrm{DC}_{n}=\bigcup_{\kappa, \tau>0} \mathrm{DC}_{n, \kappa, \tau}$. We say that $f$ is a Diophantine translation if $f(x)=x+\alpha_{f}$ for some $\alpha_{f} \in \mathrm{DC}_{d}$.

For every $\alpha \in \mathbb{R}^{d}$, let $\Theta_{\alpha, \kappa, \tau}$ be the set of all $\theta \in \mathbb{R}$ such that $(\alpha, \theta) \in \mathrm{DC}_{d+1, \kappa, \tau}$. Notice that $\Theta_{\alpha, \kappa, \tau}$ is $\mathbb{Z}$ invariant. Let $\Theta_{\alpha}=\bigcup_{\kappa, \tau>0} \Theta_{\alpha, \kappa, \tau}$. Then $\Theta_{\alpha}=\varnothing$ if $\alpha \notin \mathrm{DC}_{d}$ and $\Theta_{\alpha}$ has full Lebesgue measure if $\alpha \in \mathrm{DC}_{d}$. Moreover, every $\theta \in \Theta_{\alpha}$ is a Lebesgue density point of $\Theta_{\alpha, \kappa, \tau}$ for some $\kappa, \tau>0$.

Theorem $9([\overline{\mathrm{H}}]$, Corollaire 3, page 493 and Remarque 1, page 495). For every $\theta \in \mathbb{R}$ and $\kappa, \tau>0$, there exists a neighborhood $\mathcal{W} \subset C^{\infty}\left(\mathbb{T}^{d}, \mathrm{SL}(2, \mathbb{R})\right)$ of $R_{\theta}$ such that if $A \in \mathcal{W}$, and $\rho(f, A) \in \Theta_{\alpha_{f}, \kappa, \tau} / \mathbb{Z}$, then $(f, A)$ is $C^{\infty}$ conjugate to a constant rotation.

Proof of Theorem 8 Let $\Theta=\Theta_{\alpha_{f}}$. Since $\alpha_{f} \in \mathrm{DC}_{d}, \Theta$ has full Lebesgue measure. Let $V, E_{0}$ and $\theta$ be as in the statement of the theorem. Let $\kappa, \tau>0$ be such that $\theta$ is a Lebesgue density point of $\Theta_{\alpha_{f}, \kappa, \tau}$.

Let $\Sigma_{r}$ be the set of all $E \in \mathbb{R}$ such that $\left(f, A_{E, V}\right)$ is $C^{\infty}$ conjugate to a constant rotation.

Let $k \in \mathbb{Z}$ be such that $\rho\left(f, A_{E_{0}, V}\right)=\theta+k \alpha$. By Theorem 9 , there exists an open interval $I$ containing $E_{0}$ such that if $E^{\prime} \in I$ and $\rho\left(f, A_{E^{\prime}, V}\right)-k \alpha \in \Theta_{\alpha_{f}, k, \tau}$ then $E^{\prime} \in \Sigma_{r}$. Let $\rho: I \rightarrow \mathbb{R} / \mathbb{Z}$ be given by $\rho(E)=\rho\left(f, A_{E, V}\right)-k \alpha$. If $\rho(I)=\{\theta\}$ (this cannot really happen, but we do not need this fact), then $I \subset \Sigma_{r}$. Otherwise, by continuity of the fibered rotation number, $\rho\left(I \cap \Sigma_{r}\right) \supset \rho(I) \cap \Theta_{\alpha, \kappa, \tau} / \mathbb{Z}$ has positive Lebesgue measure. Since $\rho$ is $K(E)$-Lipschitz at every $E \in \Sigma_{r}$, we conclude in any case that $\Sigma_{r}$ has positive Lebesgue measure. The result follows by Lemma 12

Acknowledgement. We thank the referee for several suggestions that improved the exposition.

\section{REFERENCES}

[AB] A. Avila, J. Bochi. A uniform dichotomy for generic $\operatorname{SL}(2, \mathbb{R})$ cocycles over a minimal base. Preprint http://arxiv.org/math.DS/0611397 To appear in Bull. Soc. Math. France.

[AD] A. Avila, D. DAMANik. Generic singular spectrum for ergodic Schrödinger operators. Duke Math. J., 130 (2005), 393-400.

[B1] J. Bochi. Genericity of zero Lyapunov exponents. Ergod. Th. Dynam. Sys., 22 (2002), 1667-1696.

[BD] M. Boshernitzan, D. DAMANik. Generic continuous spectrum for ergodic Schrödinger operators. To appear in Commun. Math. Phys.

[B2] J. Bourgain. Positive Lyapounov exponents for most energies. In Geometric Aspects of Functional Analysis, Lecture Notes in Math. 1745 (2000), 37-66.

[B3] J. Bourgain. On the spectrum of lattice Schrödinger operators with deterministic potential. J. Anal. Math., 87 (2002), 37-75.

[B4] J. Bourgain. Green's Function Estimates for Lattice Schrödinger Operators and Applications. Annals of Mathematics Studies, 158. Princeton University Press, Princeton, 2005.

[BGS] J. Bourgain, M. Goldstein, W. Schlag. Anderson localization for Schrödinger operators on $\mathbb{Z}$ with potentials given by the skew-shift. Commun. Math. Phys., 220 (2001), 583-621.

[Cam] R. H. Cameron. Almost periodic properties of bounded solutions of linear differential equations with almost periodic coefficients. J. Math. Phys. Massachusetts 15, 73-81 (1936).

[C] N. D. Cong. A generic bounded linear cocycle has simple Lyapunov spectrum. Ergod. Th. Dynam. Sys., 25 (2005), 1775-1797.

[CF] N. D. Cong, R. FABbri. On the spectrum of the one-dimensional Schrödinger operator. Discrete Contin. Dyn. Syst., 9 (2008), 541-554.

[EJ] R. Ellis, R. A. Johnson. Topological dynamics and linear differential systems. J. Differential Equations, 44 (1982), 21-39.

[FJ] R. FAbBri, R. Johnson. Genericity of exponential dichotomy for two-dimensional differential systems. Ann. Mat. Pura Appl., 178 (2000), 175-193. 
[FJP] R. Fabbri, R. Johnson, R. Pavani. On the nature of the spectrum of the quasi-periodic Schrödinger operator. Nonlinear Anal. Real World Appl., 3 (2002), 37-59.

[F] A. Furman. On the multiplicative ergodic theorem for uniquely ergodic systems. Ann. Inst. H. Poincaré Probab. Statist., 33 (1997), 797-815.

[GJ] A. GoRDon, S. JitomiRsKaYA. In preparation.

[H] M.-R. Herman. Une méthode pour minorer les exposants de Lyapounov et quelques exemples montrant le caractère local d'un théorème d'Arnol'd et de Moser sur le tore de dimension 2. Comment. Math. Helv., 58 (1983), 453-502.

[J] R. Johnson. Exponential dichotomy, rotation number, and linear differential operators with bounded coefficients. J. Differential Equations, 61 (1986), 54-78.

[KSS] O. Kozlovski, W. Shen, S. van Strien. Density of hyperbolicity in dimension one. To appear in Ann. of Math.

[L] D. Lenz. Singular spectrum of Lebesgue measure zero for one-dimensional quasicrystals. Commun. Math. Phys., 227 (2002), 119-130.

[R] W. Rudin. Fourier Analysis on Groups. Interscience Publishers, New York-London, 1962.

[S] B. Simon. Bounded eigenfunctions and absolutely continuous spectra for one-dimensional Schrödinger operators. Proc. Amer. Math. Soc., 124 (1996), 3361-3369.

[Y] J.-C. Yoccoz. Some questions and remarks about SL $(2, \mathbb{R})$ cocycles. In Modern Dynamical Systems and Applications, 447-458. Cambridge University Press, Cambridge, 2004.

CNRS UMR 7599, Laboratoire de Probabilités et Modèles aléatoires. Université Pierre et Marie Curie-Boîte courrier 188. 75252-Paris Cedex 05, France

Current address: IMPA, Rio de Janeiro, Brazil

URL: www.proba. jussieu.fr/pageperso/artur/

E-mail address: artur@math.sunysb.edu

Instituto de Matemática, UFRGS, Porto Alegre, Brazil

Current address: Departamento de Matemática, PUC-Rio, Rio de Janeiro, Brazil

URL: www.mat.ufrgs.br/ ${ }^{\sim}$ jairo

E-mail address: jairo@mat.ufrgs.br

Department of Mathematics, Rice University, Houston, TX 77005, USA

URL: www.ruf.rice.edu/ dtd3

E-mail address: damanik@rice.edu 